Commentary

\title{
Deconstructing then Priming Gut Microbiota Resilience
}

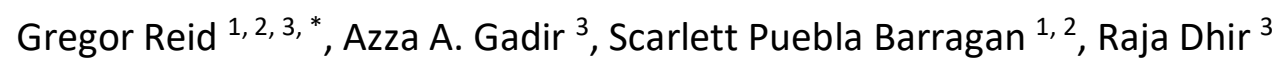

1. Canadian R\&D Centre for Human Microbiome and Probiotics, Lawson Health Research Institute, London, Canada; E-Mails: gregor@uwo.ca; spueblab@uwo.ca

2. Departments of Microbiology and Immunology, and Surgery, Western University, London, Ontario, Canada.

3. Seed Health, Venice, California, USA; E-Mails: azza@seed.com; raja@seed.com

* Correspondence: Gregor Reid; E-Mail: gregor@uwo.ca

Academic Editor: Rostyslav Bubnov

Special Issue: Gut Microbiota and Gut Health

OBM Hepatology and Gastroenterology

2021, volume 5 , issue 1

doi:10.21926/obm.hg.2101055
Received: December 11, 2020

Accepted: January 25, 2021

Published: February 01, 2021

\begin{abstract}
The human intestinal microbiota represents a complex microbial community that plays an essential role in the maintenance of host health. Over the last decade, metagenomic and metabolomic analyses have revealed the influence of intestinal microbial diversity and composition on a range of biological functions in the host. While overall taxonomic composition of the intestinal microbiome is informative, changes in spatial dynamics within the community also have profound biological significance as microbial functions are influenced by neighbouring community members and the microenvironment. Critical gaps remain in our understanding of microbiota structure, co-dependences, resilience and response to antimicrobial agents. In this review, we discuss alternative strategies to deconstruct the microbiome to yield better designed and more clinically effective therapies.
\end{abstract}

\section{Keywords}

Gut microbiota structure; co-dependences; disruptive therapies

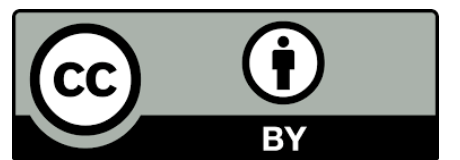

(C) 2021 by the author. This is an open access article distributed under the conditions of the Creative Commons by Attribution License, which permits unrestricted use, distribution, and reproduction in any medium or format, provided the original work is correctly cited. 


\section{Background}

The microbes that inhabit humans are increasingly recognized for a multitude of activities that benefit their eukaryotic host. While no single microbiota composition has been identified as universally healthy, there are health-conferring functions expressed directly and indirectly by different microbial compositions, particularly those of the gastrointestinal tract. A reliance on sequencing technologies to map the gut microbiota fails to capture species to species and strain to strain alliances and bacterial communication networks that may be critical for conveyance of health. This despite attempts using metagenomics that show strain and pathway co-abundances [1]. Therefore, major gaps remain in our understanding of microbial structural and metabolic symbiosis that occur within co-dependent microbial consortia at baseline and in response to antimicrobial exposure. This article will discuss these gaps, propose some methods to fill them, and encourage further research to understand how microbes connect specifically with each other. In the rush to apply fecal microbiota transplant and other interventions to influence the gut ecosystem and distant sites affected by the intestinal organisms and their by-products, we may be failing to apply specific attributes needed by the recipients and absent in the donor's stool or probiotic product.

\section{Metabolic Associations within the Gut Microbiota and Exposure to Antimicrobials}

Due to the invasive nature of gut mucosal sampling, we know less about co-aggregated pairings and mutualistic associations in the gastrointestinal tract compared to more readily accessible body sites like the oral cavity [2]. Metabolic associations and microscopy have revealed inter-linkages and glycocalyx matrix-binding organisms together in 'microniches' that specifically colonize different parts of the intestine $[3,4]$. We have yet to identify the molecular function of these microniches within a co-dependent microbial linkage, biofilm consortia or exopolysaccharide matrix.

Further, the pleiotropic activities of environmentally introduced antimicrobial compounds on the gut microbiota remain poorly understood. Rather than only considering antibiotics, the role of other compounds that disrupt the microbiome should be further investigated. These could include food additives such as sodium benzoate, sodium nitrite, and potassium sorbate [5], environmental toxins [6], numerous unmetabolized small molecules [7], as well as by constituents producing bacteriocins, host-derived antimicrobial peptides or from slgA. Empirically, clinical assessment of patients exposed to prescribed antibiotics has revealed some mutualistic activities and how single organisms and biofilms respond to different stressors. But, so far these have not interrogated the microbiota structure.

Only a few co-dependences have been described to date. For example strains of Bifidobacterium bifidum, which act as donors of fucose and lactose to allow the growth of $B$. breve (the beneficiary), and different species (Bacteroides ovatus, Bifidobacterium longum subspecies longum, Megasphaera elsdenii, Ruminococcus gnavus, Veillonella parvula) preferentially utilizing mixtures of plant polysaccharides (arabinoxylan, xyloglucan, $\beta$-glucan, pectin) [8, 9]. In vitro coaggregation studies were an early attempt to identify pairings [2], but strains may mutually benefit through mechanisms other than coaggregation. Furthermore, while surface structures and thermodynamic interactions can partly explain how coaggregation occurs, it is not clear what certain strains gain from binding to another except for retention in a given biofilm or niche, or gaining access to genetic material from each other. This is not only pertinent to the oral and intestinal sites, but also at others 
such as the skin [10] and urogenital tract [11]. This is important when designing an intervention that can stop pathogenesis. Should the microbes being administered coaggregate with pathogens to negate their virulence or disrupt coaggregates to help remove the pathogens from the niche? Both options require different approaches to selecting the intervention. Yet neither have been considered in any fecal microbiota transplantations or application of probiotics to the gut.

One way to begin to deconstruct these associations would be to identify bacterial species consistently found beside each other in stool sample sections studied by microscopy (as discussed later); then, studying some of these pairings to assess nutritional, genetic and metabolic interactions between them.

Synthetic ecosystem modelling techniques are evolving that examine how growth rates influence metabolic competition and output [12], which may be used alongside mini-chemostat or transwell models to inform temporal community dynamics in the gut biofilm to the point that outcomes could be predicted. By necessity, untargeted metabolomics are vital to detect and quantify small molecules produced individually and collectively in naïve microbial consortia and in response to challenge.

\section{Biofilm Structure and Proposed Approach}

In addition to gut microbiota composition and metabolomic output, it would be clinically beneficial to ascertain microbial co-dependences and biofilm structure. Thirteen years ago, Swidsinski et al [13] were among the first to use fluorescent in situ hybridization (FISH) probes on colonoscopic biopsies to identify bacterial species and how antibiotic therapy affected adherent organisms in the intestinal biofilm. Distal to the gut, FISH has also been applied to vaginal cells and superimposed to 400x magnification for reconstructing how Atopobium vaginae and Gardnerella vaginalis organize within a mutualistic pairing. Methods that combine fluorescent probes with high resolution magnification could similarly offer novel insight into the spatial dynamics within bacterial networks in the intestinal mucosa. Even though FISH is currently limited by the number of gut microbiota species that can be detected and fluorescent colours that can be clearly identified in a polymicrobial community, the data could help validate new therapies for certain gastrointestinal disorders. For example, tagging Bacteroides, Ruminococcus, Eubacterium rectale-Clostridium coccoides, Fusobacterium prausnitzii, Prevotella, Roseburia and Enterobacteriaceae [14] in inflammatory bowel disease in response to a specific treatment can be used for preclinical discovery and validation, or to stratify patients into responders vs. non-responders for current therapies that target gut microbiota composition and function.

Combinatorial imaging techniques may also be used to examine co-aggregated pairings in the intestinal lumen via stool sampling. Assuming even microbiota distribution within a 1gram fecal pellet, future clinical studies can conduct multiple analyses by splitting the samples into three pieces. One section could be analysed using a range of FISH probes to identify the structure and location of species. Swidsinski et al. [15] applied this method to examine the spatial arrangement of bacteria on the intestinal mucosa using three colours on a single frame. A hyperspectral imager fitted to the microscope is worth considering $[16,17]$ to provide hyperspectral data cubes with high resolution and spatial dimensions, with light covering the visible and near-infrared range from 500 to $1000 \mathrm{~nm}$; though this technique has not yet been explored for microbiota studies. Oil immersion magnification (1000x) would allow for easier differentiation of bacteria using colours. Other image 
analysis techniques will identify where species are positioned and what co-dependences might exist. Fiji ImageJ software has been used to identify biofilms on the intestinal mucosa, though limited by the use of single staining for all bacterial types [18]. Further, BiofilmQ allows for the visualization of 3D binary data dissected into cubes for spatial distribution of the organisms [19]. The use of FISH probes from shotgun reads is a new method recently reported to identify microbes and image their spatial structure [20], as these probes could hybridize to OTUs to generate confocal images on paraformaldehyde-fixed activated samples. This allows for the visualization of novel and low abundant OTUs.

A second section of fecal pellet could be examined under transmission electron microscopy either by immunostaining cryostat sections prior to embedding or by first embedding in a hydrophobic resin to generate ultrathin sections before immunogold labelling [21]. Although this technique limits the diameter of the section being examined, structural visualization of cell wall associated pili, lipopolysaccharides, microbial antigens, and bacterial entry into eukaryotic cells may provide insight into the structure and connectivity of these organisms.

A third fecal section can be analysed by metagenomic sequencing [22], combined with liquid chromatography - mass spectrometry to assess metabolic output [23]. This would confirm the presence of bacterial species and the net metabolic output, which can inform future studies to evaluate metabolomic output as a consequence of microbial co-dependences. After identifying bacterial type and abundance, additional FISH probes could be made to re-examine the sectioned material.

The outlined combinatorial approach would provide information on the organisms that are present, their genetic capabilities and functional output within the pellet, and the structured organization within the biofilm (Figure 1). The application of these methods on hundreds of samples would thereby identify key factors in resilience as well as recalcitrant biofilm structures more often associated with disease.

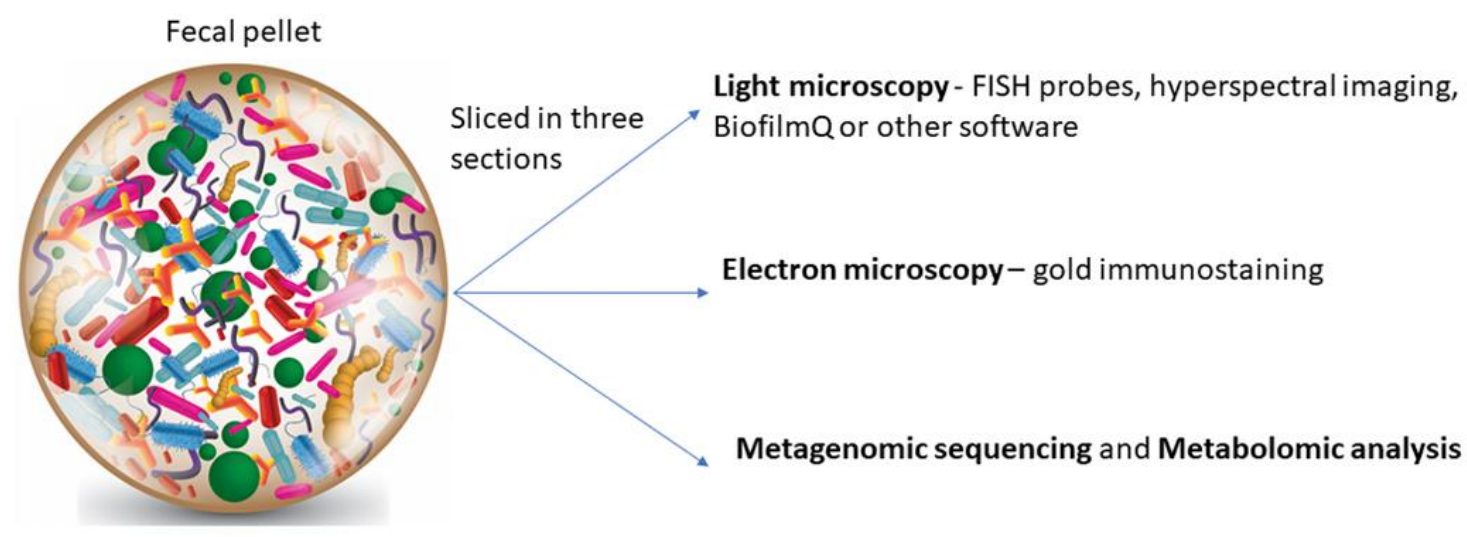

Figure 1 This combinatorial approach will inform bacterial composition and genetic capabilities, structure of the biofilm, potential co-dependencies and metabolic output.

It can be argued that stool does not represent what is happening at the tissue surfaces and indeed that different sites along the gastrointestinal tract will harbour different organisms and associations. Until a sampling method, such as a pill that can be guided to a site then able to extract material, is created that does not require intestinal content evacuation, we will have to rely on 
surgically removed tissue to identify microbes and their associations on the epithelium. Whether implanted fistula or ileal conduit sampling can offer some insight remains to be seen.

Studies on natural and synthetic microbial ecosystems have emphasized that declining biodiversity alters ecosystem behaviour, and that the nature of co-dependences and disruptive events, including those resulting from antimicrobial exposure, will influence functional outcome [24]. In response to certain genera depletion, the microbiota may allow other genera to compensate for the depleted function while still maintaining the overall metabolic output [25]. By knowing the recovery debt (deficit in biodiversity and function), it may be possible to determine how to hasten it [26].

\section{Applications of Clinical Relevance}

By understanding the composition of the microbiota, and perhaps more importantly the structure, co-dependences and resilience, experiments could be performed post- challenge with antibiotics. This would assess alteration or recovery through functional redundancy [27]. There are several advantages of the above approach, including real-time information acquired on a noninvasively collected human sample, and assessment of whether a specific pathogen within the microbiota has been eradicated or dislodged.

Biofilm stabilization may serve a purpose for therapies which induce off-target antimicrobial damage. In order to enhance such stabilization, substances such as substrates that 'feed' the biofilm, might be considered. Prebiotic compounds are substrates that are selectively utilized by host microorganisms and have potential use in stimulating recovery to a homeostatic state [28]. For example, if Akkermansia muciniphila or Bifidobacterium located on the luminal side of a biofilm, associated with a healthy state, are depleted by an antimicrobial substance, subsequent administration of a prebiotic may hasten their resilience and recovery. A novel prebiotic Lycium barbarum polysaccharide that stimulates Akkermansia along with immune modulators including slgA in the colon of mice has recently been identified [29]. The use of preferential substrates to prime gut microbiota resilience after antimicrobials deserves further investigation and will help to add to a previous attempt to create a structural model of how gut microbes interlink [30].

For the most part, the dietary components that should be administered to support resilience and functional redundancy have not been sufficiently considered in treatment and recovery of patients with antimicrobial induced biofilm disruption. If the tipping point between health and disease is an outgrowth of a dense polymicrobial pathogen biofilm [31], then dietary interventions that support competitor organisms or deprive the pathogens of key nutrients may be worth exploring. Such alignment of nutrients consumed by the host could also be merited after fecal microbiota transplant to help retain the donor organisms and health of the recipient.

Appreciating that bacteria in the gut form biofilms, it becomes possible to consider how to integrate health-promoting bacteria into the niche or use them to disrupt a formation that is detrimental to the host. This concept has been shown to be feasible in vaginal biofilms disrupted by probiotic lactobacilli [32]. An extensive review of the concept [33] suggested numerous mechanisms whereby probiotic bacteria could affect the formation of biofilms, including interfering with quorum sensing and producing antagonistic substances like acid, bacteriocins and biosurfactants that alter the surface tension and co-adhesion forces keeping the biofilm structure in place [34, 35]. 
Use of microbial monotherapy or consortia that integrate into host biofilms and perform lost function temporarily may provide a means to reduce the burden of recovery debt, until the system recalibrates. Use of the microscopy methods described above will bring us closer to understanding these mechanisms by which certain probiotics may prevent antibiotic-associated diarrhea [36].

An example of the applications we foresee: if a network of say six associated strains is linked in a biofilm and produces $p$-cresol toxin, then the clinical manipulation or intervention would have to disrupt that network so that the $\mathrm{p}$-cresol was not produced or in lower amounts. Thus, a probiotic strain would be selected for its disruptive ability of those six strains and a prebiotic would help propagate the probiotic or other similar indigenous strains of that probiotic species. The read-out would be less $p$-cresol and on examination of the feces, the six-strain network would be less abundant. These types of changes to organism connectivity could become an important driver for future therapy. Many chronic conditions that are difficult to treat may need to have specific networks broken to stop the inflammatory processes or production of toxins from continually damaging the host. Once a method is created to identify linkages between species, as this paper proposes, these types of diagnostics and interventions become feasible.

While antibiotic use comprises the majority of pharmacologically induced microbial dysbiosis, other environmental contributors need to be better understood. The Center for Disease Control has proposed the concept of One Health to interrogate the cycling of microbial communities between soil, water, agriculture, and animals as it relates to human health [37]. Many accidental stressors can have downstream effects on humans, not solely through chemical toxicity [38]. Future clinical protocols could include examining the gut microbiota composition and structure to gauge resilience against compounds with antimicrobial attributes for which the patient has been, or will be, exposed. The aforementioned methods to quantify and counter disruption in environmentally induced biofilm microbial disruptions may offer further understanding and potential therapeutic interventions.

\section{Conclusions}

A new wave of gut microbiome studies has progressed from descriptive to functional to translational. Nonetheless, the rational design of therapies to alter the resident gut microbiota will be slow and imprecise without a greater understanding of microbial co-dependences, pairings, and aggregations in relation to a human host. Employing methods to quantify the taxonomic and functional effects of environmental, pharmacological, and dietary perturbations to gut microbial linkages will help inform public health recommendations. A deeper understanding of codependences and microbial interlinkages can also improve the efficacy of prospective therapies currently in human clinical trials that are targeting the gut microbiota to treat a range of metabolic, inflammatory, immunological, and neurological conditions. For example, the same donor providing feces for transplantation to resolve chronic infection may prove inappropriate for patients with bloating, inflammation or mental illness because the microbial associations are not aligned to produce the required metabolites or reactions.

Once mechanisms of co-aggregation and co-dependency are identified, microbes with genes that increase consortia resilience could be administered or the growth of existing members could be enhanced prophylactically without fear of promoting drug resistance. 


\section{Author Contributions}

The manuscript was conceived and drafted by GR, reviewed and edited by $A G, S P B$ and RD.

\section{Competing Interests}

All authors have affiliations with Seed a company that produces a symbiotic that is not discussed herein.

\section{References}

1. Chen L, Collij V, Jaeger M, van den Munckhof IC, Vila AV, Kurilshikov A, et al. Gut microbial coabundance networks show specificity in inflammatory bowel disease and obesity. Nat Commun. 2020; 11: 1-12.

2. Rickard AH, Gilbert P, High NJ, Kolenbrander PE, Handley PS. Bacterial coaggregation: An integral process in the development of multi-species biofilms. Trends Microbiol. 2003; 11: $94-$ 100.

3. Costerton JW, Rozee KR, Cheng KJ. Colonization of particulates, mucous, and intestinal tissue. Prog Food Nutr Sci. 1983; 7: 91-105.

4. Pérez-Cobas AE, Gosalbes MJ, Friedrichs A, Knecht $H$, Artacho A, Eismann $K$, et al. Gut microbiota disturbance during antibiotic therapy: A multi-omic approach. Gut. 2013; 62: 15911601.

5. Hrncirova L, Machova V, Trckova E, Krejsek J, Hrncir T. Food preservatives induce Proteobacteria dysbiosis in human-microbiota associated Nod2-deficient mice. Microorganisms. 2019; 7: 383.

6. Iszatt N. Janssen S, Lenters V, Dahl C, Stigum H, Knight R et al. Environmental toxicants in breast milk of Norwegian mothers and gut bacteria composition and metabolites in their infants at 1 month. Microbiome. 2019; 7: 1-14.

7. aus der Beek T, Weber FA, Bergmann A, Hickmann S, Ebert I, Hein A, Kuster A. Pharmaceuticals in the environment: Global occurrences and perspectives. Environ Toxicol Chem. 2016; 35: 823835.

8. Centanni M, Ferguson SA, Sims IM, Biswas A, Tannock GW. Bifidobacterium bifidum ATCC 15696 and Bifidobacterium breve 24b metabolic interaction based on 2'-O-fucosyl-lactose studied in steady-state cultures in a Freter-style chemostat. Appl Environ Microbiol. 2019; 85: e02783-18.

9. Liu Y, Heath AL, Galland B, Rehrer N, Drummond L, Wu XW, et al. Substrate use prioritization by a coculture of five species of gut bacteria fed mixtures of arabinoxylan, xyloglucan, $\beta$-glucan, and pectin. Appl Environ Microbiol. 2020; 86: e01905-19.

10. Kumar KV, Pal A, Bai P, Kour A, Sheeba E, Rajarajan P, et al. Co-aggregation of bacterial flora isolated from the human skin surface. Microb Pathog. 2019; 135: 103630.

11. Reid G, McGroarty JA, Angotti R, Cook RL. Lactobacillus inhibitor production against Escherichia coli and coaggregation ability with uropathogens. Can J Microbiol. 1988; 34: 344-351.

12. Scott SR, Din MO, Bittihn P, Xiong L, Tsimring LS, Hasty J. A stabilized microbial ecosystem of self-limiting bacteria using synthetic quorum-regulated lysis. Nat Microbiol. 2017; 2: 1-9. 
13. Swidsinski A, Loening-Baucke V, Bengmark S, Scholze J, Doerffel Y. Bacterial biofilm suppression with antibiotics for ulcerative and indeterminate colitis: Consequences of aggressive treatment. Arch Med Res. 2008; 39: 198-204.

14. Buret AG, Motta JP, Allain T, Ferrez J, Wallace JL. Pathobiont release from dysbiotic gut microbiota biofilms in intestinal inflammatory diseases: A role for iron? J Biomed Sci. 2019; 26: 1-14.

15. Swidsinski A, Weber J, Loening-Baucke V, Hale LP, Lochs H. Spatial organization and composition of the mucosal flora in patients with inflammatory bowel disease. J Clin Microbiol. 2005; 43: 3380-3389.

16. Gao L, Smith RT. Optical hyperspectral imaging in microscopy and spectroscopy-A review of data acquisition. J Biophotonics. 2015; 8: 441-456.

17. Jansen-Winkeln B, Holfert N, Kohler H, Moulla Y, Takoh JP, Rabe SM, et al. Determination of the transection margin during colorectal resection with hyperspectral imaging (HSI). Int J Colorectal Dis. 2019; 34: 731-739.

18. Yang J, Yang $Y$, Ishii $M$, Nagata $M, A w ~ W, ~ O b a n a ~ N$, et al. Does the gut microbiota modulate host physiology through polymicrobial biofilms? Microbes Environ. 2020; 35: ME20037.

19. Hartmann R, Jeckel H, Jelli E, Singh PK, Vaidya S, Bayer M, et al. BiofilmQ, a software tool for quantitative image analysis of microbial biofilm communities. BioRxiv. 2019; 735423.

20. Tan SM, Yung PY, Hutchison PE, Xie C, Teo GH, Ismail MH, et al. Primer-free FISH probes from metagenomics/metatranscriptomics data permit the study of uncharacterised taxa in complex microbial communities. NPJ Biofilms Microbiomes. 2019; 5: 17.

21. Jones JC. Pre- and Post-embedding immunogold labeling of tissue sections. Methods Mol Biol. 2016; 1474: 291-307.

22. Basolo A, Hohenadel M, Ang QY, Piaggi P, Heinitz S, Walter $M$, et al. Effects of underfeeding and oral vancomycin on gut microbiome and nutrient absorption in humans. Nat Med. 2020; 26: 589-598.

23. Zhao L, Lou H, Peng $Y$, Chen S, Zhang Y, Li X. Comprehensive relationships between gut microbiome and faecal metabolome in individuals with type 2 diabetes and its complications. Endocrine. 2019; 66: 526-537.

24. Naeem S, Hahn DR, Schuurman G. Producer-decomposer co-dependency influences biodiversity effects. Nature. 2000; 403: 762-764.

25. Morin PJ. The complexity of co-dependency. Nature. 2000; 403: 718-719.

26. Moreno-Mateos D, Barbier EB, Jones PC, Jones HP, Aronson J, Lopez-Lopez JA, et al. Anthropogernic ecosystem disturbance and the recovery debt. Nat Commun. 2017; 8: 1-6.

27. Moya A, Ferrer M. Functional redundancy-induced stability of gut microbiota subjected to disturbance. Trends Microbiol. 2016; 24, 402-413.

28. Gibson GR, Hutkins R, Sanders ME, Prescott SL, Reimer RA, Salminen SJ, et al. Expert consensus document: The International Scientific Association for Probiotics and Prebiotics (ISAPP) consensus statement on the definition and scope of prebiotics. Nat Rev Gastroenterol Hepatol. 2017; 14: 491-502.

29. Zhu W, Zhou S, Liu J, McLean RJ, Chu W. Prebiotic, immuno-stimulating and gut microbiotamodulating effects of Lycium barbarum polysaccharide. Biomed Pharmacother. 2020; 121: 109591. 
30. Sarbini SR, Rastall RA. Prebiotics: Metabolism, structure and function. Funct Food Rev. 2011; 3: 93-106.

31. Tytgat HL, Nobrega FL, van der Oost J, de Vos WM. Bowel biofilms: Tipping points between a healthy and compromised gut? Trends Microbiol. 2019; 27: 17-25.

32. McMillan A, Dell M, Zellar MP, Cribby S, Martz S, Hong E, et al. Disruption of urogenital biofilms by lactobacilli. Colloids Surf B Biointerfaces. 2011; 86: 58-64.

33. Barzegari A, Kheyrolahzadeh K, Khatibi SM, Sharifi S, Memar MY, Vahed SZ. The battle of probiotics and their derivatives against biofilms. Infect Drug Resist. 2020; 13: 659-672.

34. Reid G, Heinemann C, Velraeds M, van der Mei HC, Busscher HJ. [31] Biosurfactants produced by Lactobacillus. Methods Enzymol. 1999; 310: 426-433.

35. Younes JA, van der Mei HC, van den Heuvel E, Busscher HJ, Reid G. Adhesion forces and coaggregation between vaginal staphylococci and lactobacilli. PLoS One. 2012; 7: e36917.

36. Mekonnen SA, Merenstein D, Fraser CM, Marco ML. Molecular mechanisms of probiotic prevention of antibiotic-associated diarrhea. Curr Opin Biotechnol. 2020; 61: 226-234.

37. van Bruggen $A H$, Goss EM, Havelaar A, van Diepeningen AD, Finckh MR, Morris Jr JG. One Health-Cycling of diverse microbial communities as a connecting force for soil, plant, animal, human and ecosystem health. Sci Total Environ. 2019; 664: 927-937.

38. Tsiaoussis J, Antoniou MN, Koliarakis I, Mesnage R, Vardavas $\mathrm{Cl}$, Izotov BN, et al. Effects of single and combined toxic exposures on the gut microbiome: Current knowledge and future directions. Toxicol Lett. 2019; 312: 72-97.

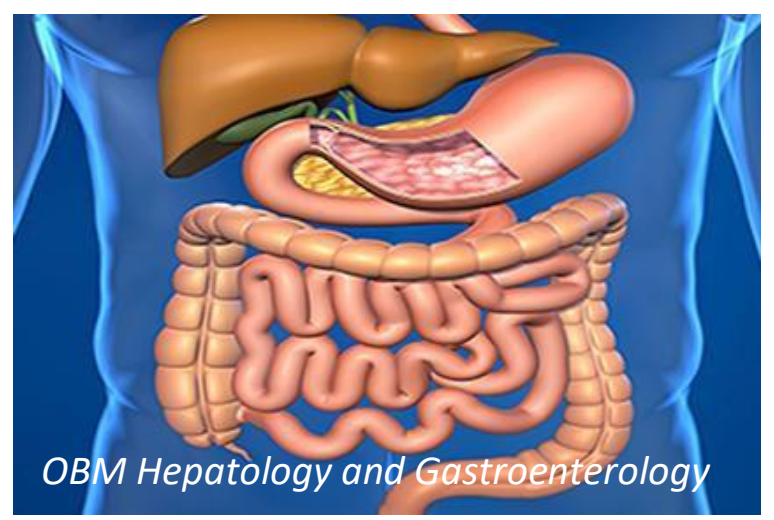

Enjoy OBM Hepatology and Gastroenterology by:

1. Submitting a manuscript

2. Joining in volunteer reviewer bank

3. Joining Editorial Board

4. Guest editing a special issue

For more details, please visit: http://www.lidsen.com/journals/hg 